relatively limited in diagnostic accuracy, leading to requirements for further investigations and uncertainty for physician and patient.

Metabolomics combines high-resolution mass spectrometry and multivariate statistical analysis, characterising thousands of compounds within a biological sample. It is ideal for biomarker discovery and elucidation of underlying disease processes, yet few studies have applied metabolomic approaches to pleural disease. However, recent pilot work has demonstrated distinct metabolomic 'features' between malignant and nonmalignant pleural effusions.

Methods 121 pleural fluid samples from a repository held at North Bristol NHS Trust underwent metabolomic analysis using flow-infusion electrospray-mass spectroscopy (FIE-MS) at the Institute of Biological, Environmental and Rural Sciences (IBERS) at Aberystwyth University. The pathologies were: mesothelioma, malignancy (all subtypes), and benign asbestos related pleural effusion (BAPE). The resultant data were analysed using the online platform Metaboanalysis 4.0. Partial Least Squares Discriminant Analysis (PLSDA) was used for feature selection based on our criteria of Variable Importance Projections (VIP) greater than 2. Data were then sub selected using these features, and analysed using Principal Component Analysis and Hierarchical Cluster Analysis (HCA). Biomarker potentials of these chosen metabolites were then assessed using AUC-ROC curves.

Results Of 108 identified features, 15 were able to differentiate BAPE from malignant effusions with AUCs of $>0.81$. The most discriminatory feature, 2-benzyl-3-iodopropanoic acid, a metabolite of uncertain clinical significance found more abundantly in malignant effusions than BAPE, was able to distinguish malignant pleural effusions from BAPE with an accuracy of $91 \%$. When combined with up to 10 other features in a panel of markers, this diagnostic accuracy increases to $98 \%$.

Conclusions We have identified many promising metabolomic features which demonstrate high accuracies in differentiating malignant effusions and BAPE. These features have the potential to be developed into future clinical diagnostic biomarkers of pleural disease, and may further our understanding of the disease processes leading to pleural effusion formation. Validation of these findings and identification of the specific metabolites is underway.

\section{S12 PAI-1 IS THE PREDOMINANT BIOLOGICAL FACTOR ASSOCIATED WITH SEPTATION FORMATION IN PLEURAL INFECTION}

${ }^{1,2,3}$ EO Bedawi, ${ }^{1,2,4} \mathrm{~N}$ Kanellakis, $4,5 \mathrm{Y}$ Zhao, ${ }^{1} \mathrm{~A}$ Sundaralingam, ${ }^{1} \mathrm{D}$ Addala, ${ }^{1,6} \mathrm{M}$ Ellayeh,
${ }^{1} \mathrm{R}$ Hallifax, ${ }^{7} \mathrm{JP}$ Corcoran, ${ }^{3} \mathrm{AM}$ Condliffe, ${ }^{1,2,4} \mathrm{NM}$ Rahman. Oxford Pleural Unit, Oxford
Centre for Respiratory Medicine, Oxford University Hospitals NHS Foundation Trust, Oxford,
UK; ${ }^{2} \mathrm{NIHR}$ Oxford Biomedical Research Centre, University of Oxford, Oxford, UK;
${ }^{3}$ Department of Infection, Immunity and Cardiovascular Disease, University of Sheffield,
Sheffield, UK; ${ }^{4}$ Laboratory of Pleural and Lung Cancer Translational Research, Nuffield
Department of Medicine, University of Oxford, Oxford, UK; ${ }^{5}$ Chinese Academy of Medical
Sciences, China Oxford Institute, Nuffield Department of Medicine, University of Oxford,
Oxford, UK; ${ }^{6}$ Department of Chest Medicine, Mansoura University, Mansoura, Egypt;
${ }^{7}$ Interventional Pulmonology Service, University Hospitals Plymouth NHS Trust, Plymouth, UK

10.1136/thorax-2021-BTSabstracts. 18
Introduction Plasminogen Activator Inhibitor-1 (PAI-1) plays an essential role in the pathogenesis of lung and pleural injury. PAI-1 levels in pleural infection have been shown to be significantly elevated compared to malignant pleural effusions and heart failure. A significant variation was seen in levels of PAI1 protein and activity in the pleural fluid from participants with pleural infection recruited to the MIST-2 study. Rabbit models of pleural injury have demonstrated that, along with other pro-inflammatory cytokines, PAI-1 is an important contributor to impaired fibrin clearance and subsequent pleural loculation. To date, this has not been studied in the context of prospectively collected pleural fluid samples from patients with confirmed pleural infection and documented baseline ultrasound septation status.

Methods Pleural fluid samples $(n=214)$ prospectively collected from patients recruited to the Pleural Infection Longitudinal OuTcomes study (PILOT) were analysed. Protein measurement assays were performed using a commercial Luminex assay for Serpin E1/PAI-1 (Luminex high performance assay, R\&D) as analyte of interest in addition to TNF-alpha, MCP-1/CCL-2, IFN-gamma, urokinase plasminogen activator (uPA) and Ddimer. The independent samples T-test was used to compare mean values for each protein between two groups (septated vs non-septated). A multinomial regression model was performed to assess the independent predictive ability for each protein to septation status as an outcome.

Results Complete ultrasound data was available for 166 cases, and these were used in the final analysis. There was a significant difference in the PAI-1 levels between the septated group $(n=122 ;$ mean $=1790.59 \mathrm{ng} / \mathrm{mL}, \mathrm{SD}=2027.28)$ and non-septated group $(\mathrm{n}=44 ;$ mean $=948.82 \mathrm{ng} / \mathrm{mL}$, $\mathrm{SD}=911.41) ; \mathrm{t}(166)=2.65, \mathrm{p}=0.009$ (Normal ref $2-46 \mathrm{ng} /$ $\mathrm{mL})$. In the multinomial regression model, PAI-1 was the only significant independent predictor of septation status $(ß=0.000, p=0.003)$.

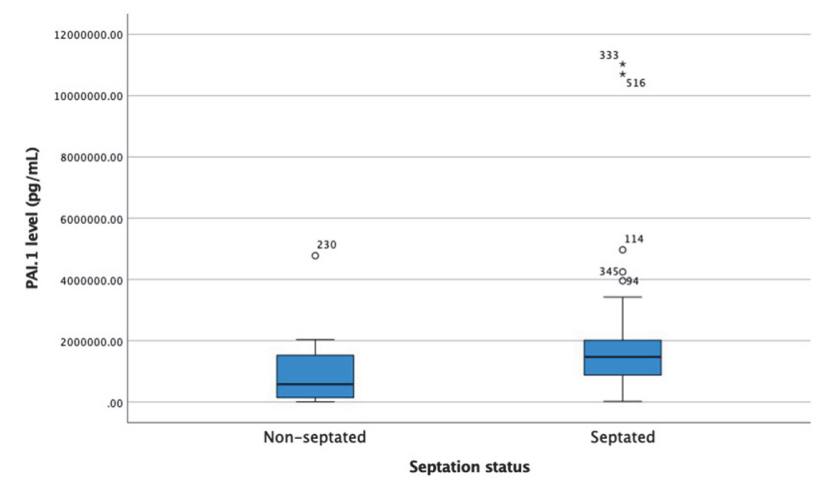

Abstract S12 Figure 1

Conclusion These data confirm that whilst several biological factors may contribute to impaired fibrinolysis and subsequent septation formation in pleural infection, PAI-1 appears to be the most important. These data imply that PAI-1 is likely to be the most useful target for further studies involving intrapleural fibrinolytic therapy in pleural infection. Further work assessing the effect of baseline PAI-1 levels on clinical outcomes in this dataset is ongoing. 